\title{
High Tissue TLR5 Expression Predicts Better Outcomes in Colorectal Cancer Patients
}

\section{Beilmann-Lehtonen, Ines}

2021-08

Beilmann-Lehtonen , I , Hagström , J , Mustonen , H K, Koskensalo , S , Böckelman , C \& Haglund, C 2021, ' High Tissue TLR5 Expression Predicts Better Outcomes in Colorectal Cancer Patients ' , Oncology , vol. 99 , no. 9 , DOI: 10.1159/000516543 , pp. 589-600 . https://doi.org/10.1159/0005

http://hdl.handle.net/10138/338687

https://doi.org/10.1159/000516543

acceptedVersion

Downloaded from Helda, University of Helsinki institutional repository.

This is an electronic reprint of the original article.

This reprint may differ from the original in pagination and typographic detail.

Please cite the original version. 


\section{High Tissue TLR5 Expression Predicts Better Outcomes in Colorectal Cancer Patients}

Ines Beilmann-Lehtonen ${ }^{1,2}$, Jaana Hagström ${ }^{2,3,4}$, Harri Mustonen ${ }^{2,5}$, Selja Koskensalo ${ }^{2,5}$, Caj Haglund $^{2,3,5, *}$, Camilla Böckelman ${ }^{2,5, *}$

${ }^{1}$ Department of Transplantation and Liver Surgery, University of Helsinki and Helsinki University Hospital, Helsinki, Finland

${ }^{2}$ Translational Cancer Medicine Research Program, Faculty of Medicine, University of Helsinki, Helsinki, Finland

${ }^{3}$ Department of Pathology, University of Helsinki and Helsinki University Hospital, Helsinki, Finland ${ }^{4}$ Department of Oral Pathology and Radiology, University of Turku, Turku, Finland

${ }^{5}$ Department of Surgery, University of Helsinki and Helsinki University Hospital, Helsinki, Finland *Equal contribution.

Running title: High tissue TLR5 predicts a better outcome in colorectal cancer patients Abbreviations: DAMP, damage-associated molecular pattern; DC, dendritic cell; DSS, diseasespecific survival; CI, confidence interval; CME, complete mesocolic excision; CRC, colorectal cancer; CRP, C-reactive protein; HR, hazard ratio; IBD, inflammatory bowel disease; IQR, interquartile range; NPC, nasopharyngeal carcinoma; NSCLC, non-small cell lung cancer; OPSCC, oropharyngeal squamous cell carcinoma; OTSCC, oral tongue squamous cell carcinoma; PAMP, pathogen-associated molecular pattern; PDAC, pancreatic ductal adenocarcinoma; SIR, systemic inflammatory response; SNP, single-nucleotide polymorphism; TLR, toll-like receptor; TMA, tissue microarray; TME, total mesorectal excision

Correspondence to:

Ines Beilmann-Lehtonen, MD

Department of Transplantation and Liver Surgery, Helsinki University Hospital 
Haartmaninkatu 4, PO Box 340

FIN-00029 HUS Finland

Phone: +358400293938

E-mail: ines.beilmann-lehtonenus.fi

Number of tables: 4

Number of figures: 3

Word count:

Keywords: toll-like receptor, colorectal cancer, colon cancer, immunohistochemistry, survival

\begin{abstract}
Background: Colorectal cancer (CRC), the third most common cancer globally, caused 881000 cancer deaths in 2018. Toll-like receptors (TLRs), the primary sensors of pathogen-associated molecular patterns (PAMPs) and damage-associated molecular patterns (DAMPs), activate innate and adaptive immune systems and participate in the development of an inflammatory tumor microenvironment. We aimed to explore the prognostic value of TLR3, TLR5, TLR7, and TLR9 tissue expressions in CRC patients.
\end{abstract}

Methods: Using immunohistochemistry, we analyzed tissue microarray (TMA) samples from 825 CRC patients who underwent surgery between 1982 and 2002 at the Department of Surgery, Helsinki University Hospital, Finland. After analyzing a pilot series of 205 tissue samples, we included only TLR5 and TLR7 in the remainder of the patient series. We evaluated the associations between TLR5 and TLR7 tissue expressions, clinicopathological variables, and survival. Using the Kaplan-Meier method, we generated survival curves, determining significance through the log-rank test. Univariate and multivariate survival analyses relied on the Cox proportional hazards model.

Results: The five-year disease-specific survival (DSS) was 55.9\% among TLR5-negative [95\% confidence interval (CI) 50.6-61.2\%] and 61.9\% (95\% CI 56.6-67.2\%; $p=0.011, \log -$ rank test) among TLR5-positive patients. In the Cox multivariate survival analysis adjusted for age, sex, stage, location, and grade, positive TLR5 immunoexpression [hazard ratio (HR) $0.74 ; 95 \%$ CI 0.59-0.92; $p$ 
$=0.007$ ) served as an independent positive prognostic factor. TLR7 immunoexpression exhibited no prognostic value in the survival analysis across the entire cohort (HR 0.97; 95\% CI 0.78-1.20; $p=$ $0.754)$ nor in subgroup analyses.

Conclusions: We show for the first time that a high TLR5 tumor tissue expression associates with a better prognosis in CRC patients.

\section{Introduction}

Colorectal cancer (CRC) is the third most commonly diagnosed malignancy in the world and the second leading cause of cancer mortality, resulting in 881000 cancer deaths in 2018 (1). The burden of CRC is increasing rapidly and 2.2 million new CRC cases are expected in $2030(1,2)$. Although CRC survival has improved in developed countries, approximately $17 \%$ of stage II and $36 \%$ of stage III patients experience recurrence within 5 years (3).

Inflammation plays a crucial role in gastrointestinal cancers, $\mathrm{CRC}$ among them, although the molecular mechanisms behind this relationship are incompletely understood. Numerous biomarkers have been investigated to reveal this connection, among them toll-like receptors (TLRs) (4-6).

TLRs are transmembranous pattern-recognition receptors (PRRs) expressed on the cell surface or intracellularly in the endosomes of innate immune cells such as macrophages, dendritic cells (DCs), neutrophils, mast cells, epithelial cells, and endothelial cells. TLRs can recognize various pathogen-associated molecular patterns (PAMPs) of a bacterial, viral, and fungal origin, and can also be activated by host-derived endogenous damage-associated molecular patterns (DAMPs), ligands released, for example, as stress responses and apoptosis (7-9).

Innate immune responses activated by TLRs are needed to evoke the immune surveillance mechanisms of the adaptive immune system $(9,10)$. In order to grow and progress, tumor cells must overcome these immune surveillance mechanisms $(10,11)$. In the same tumor, both TLR-induced protumorigenic and antitumorigenic processes can be present simultaneously $(10,12)$. 
Evidence indicating the prognostic value of TLRs in malignancies has proliferated in recent decade. For instance, a recent meta-analysis demonstrated that elevated TLR4 and TLR7 serve as markers of a poorer prognosis in several malignancies (13). In CRC patients, TLRs have not been widely investigated. In our previous study, Dukes B CRC patients with a strong TLR4 tissue expression exhibited a worse prognosis, while Dukes C CRC patients with a strong TLR2 expression experienced a better prognosis (14).

Moreover, to our knowledge, the prognostic role of TLR5 tissue immunohistochemical expression in CRC has not been previously investigated. Among other malignancies, a high TLR5 tissue expression associates with a better prognosis, for example, in non-small cell lung cancer (NSCLC) (15) and gastric cancer (16). By contrast, in HPV-positive oropharyngeal squamous cell carcinoma (OPSCC), a high TLR5 level associates with a poor outcome (17).

In this study, we aimed to study the potential prognostic role of the tissue expression of TLR3, TLR5, TLR7, and TLR9 in CRC patients.

\section{Materials and Methods}

\section{Patients}

For this study, we selected samples from 825 CRC patients who underwent surgery at the Department of Surgery, Helsinki University Hospital, Finland, between 1982 and 2002. The median age at the time of surgery was 67.5 years [interquartile range (IQR) 57.6-75.3 years] and 457 (55.4\%) of all patients were male. Clinical data were collected from patient medical records. The Population Register Center of Finland and Statistics Finland provided follow-up vital statistics data and cause of death information for deceased patients. The median follow-up time was 5.06 years (IQR 1.2-17.1), and $654(82.9 \%)$ patients died by the end of follow-up, 362 (43.9\%) from CRC. The 5-year diseasespecific survival (DSS) for all patients was 58.8\% [95\% confidence interval (CI) 55.3-62.35]. The distribution for staging from the modified Dukes classification (18), used for CRC staging in our 
clinic during patient recruitment, was as follows: 122 (14.8\%) cases were Dukes stage A, 292 (35.4 \%) Dukes B, 225 (27.3 \%) Dukes C, and 186 (22.5\%) Dukes D. Patient's clinicopathological characteristics are shown in Supplementary Table 1.

The Surgical Ethics Committee of Helsinki University Hospital (Dnro HUS 226/E6/06, extension TMK02 $\$ 66$ 17.4.2013) approved the study protocol and the National Supervisory Authority of Health and Welfare granted permission to study the archived tissue samples retrospectively without requiring individual consent (Valvira Dnro 10041/06.01.03.01/2012).

\section{Tissue samples}

Formalin-fixed and paraffin-embedded surgical tumor samples originated from the archives of the Department of Pathology, University of Helsinki. An experienced pathologist (JH) marked representative areas on hematoxylin- and eosin-stained slides. From the annotated areas, three 1.0$\mathrm{mm}$ cores were punched using a semiautomatic tissue arrayer (Beecher Instruments Inc., Silver Spring, MD, USA), and embedded in recipient paraffin tissue microarray (TMA) blocks. We cut 4$\mu \mathrm{m}$ sections from the TMA blocks for immunohistochemistry as described elsewhere (19).

\section{Immunohistochemistry}

The same immunohistochemical staining protocol was applied to each TLR. The 4- $\mu \mathrm{m}$ TMA sections were deparaffinized in xylene for $15+5 \mathrm{~min}$, rehydrated in solutions containing decreasing concentrations of ethanol, beginning with pure alcohol and ending with distilled water, for 5 min in each. For antigen retrieval, the slides were treated in a PreTreatment module (Lab Vision UK Ltd, $\mathrm{UK}$ ) in a Tris- $\mathrm{HCl}$ buffer ( $\mathrm{pH} \mathrm{8.5)} \mathrm{for} 20 \mathrm{~min}$ at $98^{\circ} \mathrm{C}$. We stained the slides in an Autostainer 480 (Lab Vision, Fremont, California, USA) using the REAL EnVision Detection System (peroxidase/DAB+, rabbit/mouse; Dako, Glostrup, Denmark). Endogenous peroxidase blocking was carried out using the $0.3 \%$ Dako REAL Peroxidase-Blocking Solution incubated for $5 \mathrm{~min}$. Then, the 
primary antibody incubation of TMA slides was carried out using the following primary antibodies: TLR3 rabbit polyclonal (sc-10740, Santa Cruz Biotechnology, Santa Cruz, CA, USA; diluted to 1:100), TLR5 mouse monoclonal (IMG-664A, Imgenex, San Diego, CA, USA; diluted to 1:200), TLR7 rabbit polyclonal (IMG-581A, Imgenex, San Diego, CA, USA; diluted to 1:300), and TLR9 rabbit polyclonal (sc-25468, Santa Cruz Biotechnology, Santa Cruz, CA, USA; diluted to 1:100). Finally, the samples were incubated with the peroxidase-conjugated Dako REAL EnVision/HRP, Rabbit/Mouse (ENV) secondary antibody for $30 \mathrm{~min}$, visualized by the Dako REAL DAB+ Chromogen for $10 \mathrm{~min}$, and counterstained with Meyer's hematoxylin and mounted in Pertex Mounting (Histolab Products AB, Sweden). In each staining series, a positive control was used from tissue known to exhibit a high immunoreactivity to the studied antigens (tonsillar, skin, and cutaneous squamous cell carcinoma tissue). As a negative control, we used specimens processed without a primary antibody.

\section{Scoring of samples}

Immunostainings of TLR3, TLR5, TLR7, and TLR9 were interpreted by two independent researchers, including an experienced pathologist from the Department of Pathology, University of Helsinki (IB-L and JH), both blinded to the clinical data. A brown cytoplasmic color in the tumor cells reflected the immunopositivity of TLR3, TLR7, and TLR9. TLR5 immunopositivity was indicated as brown staining of the nuclear membranes of the tumor cells. The immunoreactivity was scored from 0 to 3 : negative staining was scored as 0 , weak positive immunoactivity as 1 , moderate as 2, and strong as 3 (Figure 1). If the scores for a patient differed, the highest score was chosen for the final calculations. After comparing the scores from both researchers, all spots with different scoring results were re-evaluated and the final score was reached through discussion and consensus.

\section{Statistical analysis}


For the statistical analyses, the immunoexpression for each TLR in the pilot series was dichotomized into mild (0-1) and high (2-3) expression levels. In the larger cohort, TLR5 immunoexpression was dichotomized into negative (0) and positive (1-3) expression levels and TLR7 immunoexpression was dichotomized into mild (0-2) and strong (3) expression levels, based on the analysis of DSS curves of all scores (Supplemetary Figure 1). The associations between the TLR5 and TLR7 immunoexpressions and clinicopathological variables were evaluated using the chi-square test. DSS was calculated from surgery to death from CRC or until the end of follow-up. We used the KaplanMeier method to generate survival curves and the log-rank test to compare different groups. The $95 \%$ confidence intervals (CI) were calculated for survival rates.

In addition, we used the Cox proportional hazards model for the univariate and multivariate survival analyses. For the multivariate analysis, we entered the following covariates: age, gender, Dukes stage, WHO grade, tumor location, and TLR5 immunoexpression. The Dukes stage was processed as a categorical covariate. A time-dependent variable was included for Dukes stage D, tumor grade, and tumor location to fulfill the Cox's assumption. Interaction terms were considered, for which we found no significant interactions following a Bonferroni correction for multiple comparisons. All tests were two-tailed, and we considered $p<0.05$ as statistically significant. All statistical analyses were performed using SPSS version 24.0 (IBM's SPSS Statistics, version 24.0 for Mac; SPSS, Inc., Chicago, IL, USA) and SPSS version 25.0 (IBM's SPSS Statistics, version 25.0 for Mac; SPSS, Inc., Chicago, IL, USA).

\section{Results}

\section{Pilot staining}

The immunostainings for TLR3, TLR5, TLR7, and TLR9 were initially completed on a smaller cohort of 205 CRC patients. Among that cohort, we successfully scored the TLR3 immunostaining for $198(96.6 \%)$ samples, TLR5 for $166(81 \%)$, TLR7 for $199(97.1 \%)$, and TLR9 for 196 (95.6\%) 
samples. Some samples were lost due to technical reasons, such as an inadequate position of the spot or the lack of a tumor in the TMA spot. Representative images for the different TLR staining patterns appear in Figure 1.

For statistical analysis from the pilot series, the immunoexpression of each TLR was grouped into mild (scores 0-1) and strong (scores 2-3) expression levels. In the Kaplan-Meier analysis of the pilot cohort, we found no differences in prognosis between the different TLR3 (HR 0.91; 95\% CI 0.55-1.51; $p=0.722$ ), TLR5 (HR 0.66; 95\% CI 0.93-1.12; $p=0.126$ ), TLR7 (HR 0.76; 95\% CI $0.48-1.21 ; p=0.245$ ), and TLR9 (HR 1.38; 95\% CI 0.432-4.37; $p=0.589$ ) immunoexpression groups (Figure 2). We then continued examining TLR5 and TLR7 expressions in a larger study population, setting aside TLR3 and TLR9 from further analysis, as from pilot cohort DSS results we concluded that TLR5 and TLR7 were promising markers in CRC but TLR3 and TLR9 did not provide any prognostic information.

\section{Immunostaining for TLRs in a larger population}

Among 825 CRC tumor samples represented in the TMA, TLR5 immunoexpression on nuclear membranes was successfully interpreted in 734 (89\%) cases, while TLR7 cytoplasmic immunostaining was successful in $769(93.2 \%)$ samples. The detailed distribution of immunostainings appears in Table 1 . The evaluation failed in $11 \%$ of TLR 5 and $6.8 \%$ of TLR7 samples due to technical staining problems such as the folding of tissue or overlapping spots. For the statistical analyses, patients were divided into two groups consistent with the pilot series.

\section{Association with clinicopathological parameters}

An association emerged between mild TLR5 and mild TLR7 immunoexpressions and mucinous histological type $(p<0.001$ and $p=0.22$, respectively; chi-square test, Table 2). Mild TLR7 immunoexpression associated with a higher tumor grade $(p<0.001$; chi-square test, Table 2$)$ and 
Dukes stage A ( $p=0.048$; chi-square test, Table 2). No association emerged between TLR5 and TLR7 immunoexpressions with age, gender, side, or location of the primary tumor. TLR5 immonoexpression did not associate with the tumor stage.

\section{Survival analysis}

Patients with a strong TLR5 immunoexpression (HR 0.76, 95\% CI 0.61-0.93; $p=0.011$; Figure 3a and Suppl. Figure 1) exhibited a better prognosis. The 5-year DSS was 55.9\% among mild TLR5 patients (95\% CI 50.6-61.2\%) and 61.9\% (95\% CI 56.6-67.2\%; $p=0.011$, log-rank test; Figure 3a) among strong TLR5 expression patients. TLR7 immunoexpression provided no prognostic value in the survival analysis (HR 1.04, 95\% CI 0.83-1.29; $p=0.754$; Figure $3 \mathrm{~b}$ and Suppl. Figure 1).

In the subgroup analysis, patients with a left-sided tumor and a strong TLR5 immunoexpression exhibited a better prognosis (HR 0.76, 95\% CI 0.59-0.98; $p=0.035$; Figure 3d and Table 4). Also among adenocarcinoma subgroup, patients with a strong TLR5 immunoexpression showed a better survival (HR 0.73, 95\% CI 0.58-0.93; $p=0.010$; Table 4). This same trend emerged in female patients (HR 0.71, 95\% CI 0.51-0.99; $p=0.042$; Table 4) and among older patients (HR $0.67,95 \%$ CI $0.50-90 ; p=0.007$; Table 4). In the subgroup analysis for the Dukes stage with borderline significance, Dukes B patients with a strong TLR5 immunoexpression exhibited a better prognosis (HR 0.61, 95\% CI 0.37-1.00; $p=0.050$; Figure $3 \mathrm{c}$ and Table 4). TLR7 provided no prognostic value in the subgroup analysis (Table 4).

In the Cox multivariate survival analysis, in addition to age, sex, Dukes stage, and WHO grade, a strong TLR5 immunoreactivity (HR 0.074, 95\% CI 0.59-0.92; $p=0.007$ ) emerged as an independent positive prognostic factor (Table 3).

\section{Discussion}


Our findings demonstrate that a high TLR5 tissue expression may identify CRC patients with more favorable prognoses. That is, a strong TLR5 tissue expression level emerged as an independent prognostic factor. To our knowledge, this is the first study to demonstrate the prognostic role of TLR5 among CRC patients. TLR3, TLR7, and TLR9 showed no prognostic significance here.

The prognostic value of various TLRs has been studied in several malignancies. However, according to a recent meta-analysis, not very many studies had been published in this field by 2017 , with TLR4 emerging as the most widely studied TLR (13). This meta-analysis concluded that the elevated expressions of TLR4 and TLR7 predicted worse outcomes in several malignancies.

The role of TLRs in cancer development is diverse, since they can carry both tumorinducing and antitumorigenic effects (12). In vitro studies of different carcinomas confirmed that TLR5 plays a different role depending upon the malignancy. For example, TLR5 activation enhances the proliferation of gastric cancer cells (20). In contrast, knocking down TLR5- or MyD88-induced responses in mouse xenograph models of human colon adenocarcinoma resulted in tumor growth, while in flagellin-treated tumors a TLR5-induced antitumor activity was observed leading to tumor necrosis and a decrease in the tumor size (21). This finding agrees with our results, demonstrating that TLR5 might carry an antitumorigenic effect in CRC. However, previous studies relied on in vitro studies and we conducted an in vivo study, rendering these results not directly comparable.

Moreover, in a study among 70 patients with colorectal polyps, no TLR2, TLR3, TLR4, and TLR5 expression was observed in the polyps (22). Yet, TLR7 and TLR9 expressions were higher in the polyps compared to the normal colon mucosa in controls, especially in hyperplastic and adenomatous polyps, the more benign polyp types. Furthermore, within this cohort, colon cancer was diagnosed in 38 patients either before, during, or after a polypectomy. These CRC patients exhibited lower TLR7 and TLR9 expressions in their polyps, indicating an antitumorigenic role for TLR7 and TLR9 as well. Moreover, these CRC patients exhibited no TLR5 expression in the 
polyps, possibly reflecting that TLR5 may become more important following malignant development and, thus, not as relevant during the premalignant phase. However, TLR7 may play an important antitumorigenic role during the premalignant phase, possibly explaining why we found no relevant role of TLR7 in our CRC patient cohort.

In addition, the expression of genes related to TLRs were investigated using multiplex quantitative nuclease protection assay (qNPA) in 63 male CRC patients, during which the downregulation of TLR5, MyD88, IL1A, and IL6 in the tumor appeared to worsen overall survival (23). That study supports our findings of a strong TLR5 tissue immunoexpression as a positive prognostic marker. Unfortunately, that cohort was small, including only male patients without access to cause of death data for all patients, making it impossible to determine or analyze DSS.

Although the prognostic role of tumoral TLR5 has not been studied in CRC patients previously, studies on other malignancies have yielded promising results. In 313 gastric cancer patients, Kasurinen et al. examined TLR1, TLR2, TLR5, TLR7, and TLR9 tissue immunoexpressions (16). They found that patients with a high TLR5 tissue expression exhibited a better prognosis, in agreement with our findings among CRC patients. Stage III gastric cancer patients with a high TLR7 tissue expression exhibited a better DSS than patients with a low TLR7 tissue expression. Thus, Kasurinen et al. observed both nuclear and cytoplasmic expressions for all TLRs, while we observed TLR5 nuclear membrane expression and TLR7 cytoplasmic granulomatous expression, confirming that in various cancers TLRs can play different roles and exhibit different expression patterns. The antibodies used may also influence different expressions patterns. For instance, we used mouse monoclonal TLR5 antibodies and TLR7 rabbit polyclonal antibodies in our study like Kasurinen et al., although the manufacturers of the antibodies differed. In contrast to our study, among 154 pancreatic ductal adenocarcinoma (PDAC) patients, TLR5 tissue immunoexpression provided no prognostic value (24), possibly explaining the versatile 
role of TLRs in different cancers. Mouse monoclonal TLR5 and rabbit polyclonal TLR7 were used, provided by different manufacturers compared to the antibodies we used.

In oral tongue squamous cell carcinoma (OTSCC) patients, moderate and strong TLR5 tissue expressions exhibited better outcomes, although TLR5 did not serve as an independent prognostic factor (25). Interestingly, using the same antibody that we used, Mäkinen et al. observed cytoplasmic TLR5 positivity, while in our CRC patients the TLR5 staining appeared in the nuclear membrane. By contrast, our TLR7 positivity was cytoplasmic, while in the OTSCC patients, TLR7 expression appeared in the nuclear membrane. In oropharyngeal squamous cell carcinoma (OPSCC), a high TLR5 immunoexpression associated with a worse prognosis among HPV-positive tumors (17). Similar results emerged for nasopharyngeal tumors (NPC) (26), where a high TLR7 tissue expression predicted a better prognosis and patients with a strong TLR5 expression experienced a worse prognosis.

The abovementioned studies demonstrate that the TLR5 staining pattern differs and TLR5 associates with prognosis in a varying manner, depending upon the cancer type and likely on the antibody as well. The precise biology behind this remains unknown, although it is obvious that TLRs play diverging roles in different cancers $(12,27,28)$. Single-nucleotide polymorphism (SNP) within the TLR genes could partly explain these divergent roles among TLRs (29).

A TLR-targeted therapy has been studied in several malignancies including CRC and several clinical phase I and II trials are ongoing (30,31). To date, only the TLR7 agonist imiquimod has received approval for clinical use in basal cell carcinoma, used topically as a monotherapy or combined with other agents (32). Obviously, cancer immunotherapy represents a promising weapon to combat malignancies in the future. We need to identify solid biomarkers to distinguish tumors with different clinical behaviors and assist in developing targeted therapy for CRC patients. Furthermore, suitable biomarkers could help identify stage II CRC patients at a higher risk of 
recurrence and simultaneously identify stage III patients at a lower risk and, thus, spared from treatment. TLR5 tissue expression could represent a promising biomarker in the future.

The biology behind TLR activation and following cascades is very complex and still unknown. TLRs may participate in activation of metalloproteinases and cytokines, and other intracellular cascades. The TLR activation may be different depending on activation by DAMP or PAMP signals. The biological explanation for our results dealing with TLR5 expression having effect on prognosis is still unrevealed. We can only speculate that possibly, if TLR5 is not present and active the cellular cascades will stay inactivated, and this phenomenon might lead to worse prognosis. Our large and well-characterized patient material dates back several years, representing one of the strengths of our study, allowing us a long follow-up period and providing reliable subgroup analyses. Adjuvant therapy was not that common at that time, giving us a valuable opportunity to investigate the more natural course of CRC. In addition, the tumor tissue microenvironment could be remarkably affected by neoadjuvant treatment causing a bias. Using TMA slides for the analysis provided us with several advantages, by saving the primary tissue material and allowing us the opportunity to study large patient cohorts at less expense. Furthermore, the TMA technique requires an experienced specialist to minimize folding or missing spots. Thus, debate continues regarding whether TMA is sufficiently representative to rely on small $1.0-\mathrm{mm}$ tumor cores, although previous studies demonstrated that even a few cores, or even just one, could be representative of the tumor $(33,34)$. Using archival TMA blocks could be seen as disadvantage as older tissue blocks might show different staining pattern compared to younger tissue blocks. We have investigated TLR5 and TLR7 stainings in newer tissue samples, which will be published separately with other projects. We did not see impressive differences in staining patterns between older and newer tissue samples (data not shown). Also missing of validation series in this study can be criticized, but as mentioned above, we have investigated TLR5 and TLR7 stainings in newer cohort and the results are in line with the results of current study (unpublished data). 
Surgical techniques have changed in recent decades. The gold standard for colorectal surgical technique remains a total mesorectal excision (TME) for rectal tumors and complete mesocolic excision (CME) for colon tumors, both following the principles of operating along embryological planes $(35,36)$. Using these techniques, the amount of retrieved lymph nodes has increased, thereby improving the prognostic value for the outcome of CRC patients (37). Thus, the pathology reports of resected tumors have significantly improved over the years. In our cohort, unfortunately, less detailed reports were available for most patients than in the structured synoptic report that currently represents the gold standard (38), leading to a lack of histopathological information, such as perineural, vascular, and lymphatic invasion. In addition, the amount of harvested lymph nodes from a specimen impacts the bias and harvesting fewer lymph nodes can cause mistakes in the tumor staging in an older cohort (39).

\section{Conclusions}

In conclusion, we showed for the first time that a strong TLR5 tumor tissue expression could be useful in identifying CRC patients likely to enjoy a better prognosis. Specifically, a strong TLR5 tissue expression served as an independent prognostic factor. Thus, TLR5 represents a promising biomarker, and further multicenter studies are needed to validate our results.

\section{Notes}

\section{Acknowlwdgements}

The authors thank Vanessa Fueller for exceptional English-language revision, Päivi Pletokangas and Päivi Saarinen for technical assistance, and funders mentioned.

\section{Statement of Ethics}

The study protocol was approved by The Surgical Ethics Committee of Helsinki University Hospital (Dnro HUS 226/E6/06, extension TMK02 §66 17.4.2013). The National Supervisory Authority of Health and Welfare granted permission to use tissue archive samples retrospectively without requiring individual informed consent (Valvira Dnro 10041/06.01.03.01/2012). 


\section{Funding sources}

This study was supported by the Competitive State Research Financing of the Expert Responsibility Area of Helsinki University Hospital (CH), the Kurt och Doris Palander Foundation (IBL), the Finnish Cancer Foundation (CH and JH), Finska Läkaresällskapet (CB, IBL), the K. Albin Johansson Foundation (CB, IBL), and the Sigrid Juselius Foundation $(\mathrm{CH})$, and the Faculty of Medicine, University of Helsinki, Helsinki, Finland (CB, IBL)

\section{Conflict of Interst Statement}

The authors have no conflict of interest to declare.

\section{Author contributions}

IBL, CB, CH, and JH conceived and designed the study. IBL, CB, HM, and SK collected and processed the data. JH contributed to specimen preparation. IBL and JH scored the histological samples. IBL and HM analyzed the data and interpreted the results. IBL drafted the manuscript and prepared the original figures and tables. All authors revised the manuscript and provided their final approval to the current version of the submitted manuscript.

\section{References}

1. Bray F, Ferlay J, Soerjomataram I, Siegel RL, Torre LA, Jemal A. Global cancer statistics 2018: GLOBOCAN estimates of incidence and mortality worldwide for 36 cancers in 185 countries. CA Cancer J Clin. 2018 Nov;68(6):394-424.

2. Arnold M, Sierra MS, Laversanne M, Soerjomataram I, Jemal A, Bray F. Global patterns and trends in colorectal cancer incidence and mortality. Gut. 2017 Mar 11;66(4):683-91.

3. Böckelman C, Engelmann BE, Kaprio T, Hansen TF, Glimelius B. Risk of recurrence in patients with colon cancer stage II and III: a systematic review and meta-analysis of recent literature. Acta Oncol. Taylor \& Francis; 2015 Jan;54(1):5-16.

4. Fukata M, Abreu MT. Role of Toll-like receptors in gastrointestinal malignancies. Oncogene. 2008 Jan 7;27(2):234-43.

5. Klampfer L. Cytokines, inflammation and colon cancer. Curr Cancer Drug Targets. 2011 May;11(4):451-64.

6. Kim ER. Colorectal cancer in inflammatory bowel disease: The risk, pathogenesis, prevention and diagnosis. WJG. 2014;20(29):9872-11.

7. Takeda K. Toll-like receptors in innate immunity. International Immunology. 2004 Nov 22;17(1):1-14.

8. Chen K, Huang J, Gong W, Iribarren P, Dunlop NM, Wang JM. Toll-like receptors in inflammation, infection and cancer. Int Immunopharmacol. 2007 Oct;7(10):1271-85.

9. Rakoff-Nahoum S, Medzhitov R. Toll-like receptors and cancer. Nat Rev Cancer. Nature Publishing Group; 2009 Jan;9(1):57-63. 
10. Mantovani A, Allavena P, Sica A, Balkwill F. Cancer-related inflammation. Nature. 2008 Jul 24;454(7203):436-44.

11. Basith S, Manavalan B, Yoo TH, Kim SG, Choi S. Roles of toll-like receptors in Cancer: A double-edged sword for defense and offense. Arch Pharm Res. Pharmaceutical Society of Korea; 2012 Sep 1;35(8):1297-316.

12. Sato Y, Goto Y, Narita N, Hoon DSB. Cancer Cells Expressing Toll-like Receptors and the Tumor Microenvironment. Cancer Microenviron. Springer Netherlands; 2009 Sep;2 Suppl 1(S1):205-14.

13. Wang Q, Zhang X, Xiao T, Pan C, Liu X, Zhao Y. Prognostic role of Toll-like receptors in cancer: a meta-analysis. Ther Clin Risk Manag. Dove Press; 2018; Volume 14:1323-30.

14. Beilmann-Lehtonen I, Böckelman C, Mustonen H, Koskensalo S, Hagström J, Haglund C. The prognostic role of tissue TLR2 and TLR4 in colorectal cancer. Virchows Arch. 2020.

15. Zhou H, Chen J-H, Hu J, Luo Y-Z, Li F, Xiao L, et al. High expression of Toll-like receptor 5 correlates with better prognosis in non-small-cell lung cancer: an anti-tumor effect of TLR5 signaling in non-small cell lung cancer. J Cancer Res Clin Oncol. 2014 Feb 19;140(4):63343 .

16. Kasurinen A, Hagström J, Laitinen A, Kokkola A, Böckelman C, Haglund C. Evaluation of toll-like receptors as prognostic biomarkers in gastric cancer: high tissue TLR5 predicts a better outcome. Sci Rep. Nature Publishing Group; 2019 Aug 29;9(1):12553-10.

17. Jouhi L, Mohamed H, Mäkitie A, Remes SM, Haglund C, Atula T, et al. Toll-like receptor 5 and 7 expression may impact prognosis of HPV-positive oropharyngeal squamous cell carcinoma patients. Cancer Immunol Immunother. 4 ed. Springer Berlin Heidelberg; 2017 Dec;66(12):1619-29.

18. Dukes C. The classification of cancer of the rectum. J Pathol Bacteriol. 1932; 35: 323.

19. Kallioniemi OP, Wagner U, Kononen J, Sauter G. Tissue microarray technology for highthroughput molecular profiling of cancer. Hum Mol Genet. 2001 Apr;10(7):657-62.

20. Park J-H. Flagellin promotes the proliferation of gastric cancer cells via the Toll-like receptor 5. International Journal of Molecular Medicine. 2011 Mar 23;:1-5.

21. Rhee SH, Im E, Pothoulakis C. Toll-Like Receptor 5 Engagement Modulates Tumor Development and Growth in a Mouse Xenograft Model of Human Colon Cancer. Gastroenterology. 2008 Aug;135(2):518-528.e3.

22. Eiró N, González L, González LO, Andicoechea A, Fernández-Díaz M, Altadill A, et al. Study of the Expression of Toll-Like Receptors in Different Histological Types of Colorectal Polyps and Their Relationship with Colorectal Cancer. J Clin Immunol. 2012 Feb 29;32(4):848-54.

23. Royse KE, Chen L, Berger DH, Ittmann MM, El-Serag HB, Balentine CJ, et al. Expression of pattern recognition receptor genes and mortality in patients with colorectal adenocarcinoma. Int J Mol Epidemiol Genet. e-Century Publishing Corporation; 2017;8(2):8-18. 
24. Lanki M, Seppänen H, Mustonen H, Hagström J, Haglund C. Toll-like receptor 1 predicts favorable prognosis in pancreatic cancer. Canzian F, editor. PLoS ONE. Public Library of Science; 2019;14(7):e0219245.

25. Mäkinen LKM, Atula T, Häyry V, Jouhi L, Datta N, Lehtonen S, et al. Predictive role of tolllike receptors 2, 4, and 9 in oral tongue squamous cell carcinoma. Oral Oncol. Elsevier Ltd; 2015 Jan 1;51(1):96-102.

26. Ruuskanen M, Leivo I, Minn H, Vahlberg T, Haglund C, Hagström J, et al. Expression of toll-like receptors in non-endemic nasopharyngeal carcinoma. BMC Cancer. 4 ed. 2019 Jun 25;19(1):335-12.

27. Pradere J-P, Dapito DH, Schwabe RF. The Yin and Yang of Toll-like receptors in cancer. Oncogene. 2013 Aug 12;33(27):3485-95.

28. Dajon M, Iribarren K, Cremer I. Toll-like receptor stimulation in cancer: a pro- and antitumor double-edged sword. Immunobiology. 2017;222.

29. Okazaki S, Stintzing S, Sunakawa Y, Cao S, Zhang W, Yang D, et al. Predictive value of TLR7polymorphism for cetuximab-based chemotherapy in patients with metastatic colorectal cancer. Int J Cancer. 2017 Jun 21;141(6):1222-30.

30. Smith M, García-Martínez E, Pitter MR, Fucikova J, Spisek R, Zitvogel L, et al. Trial Watch: Toll-like receptor agonists in cancer immunotherapy. OncoImmunology. Taylor \& Francis; 2018;7(12):e1526250.

31. Moradi-Marjaneh R, Hassanian SM, Fiuji H, Soleimanpour S, Ferns GA, Avan A, et al. Toll like receptor signaling pathway as a potential therapeutic target in colorectal cancer. J Cell Physiol. 2018 Aug;233(8):5613-22.

32. Kamath P, Darwin E, Arora H, Nouri K. A Review on Imiquimod Therapy and Discussion on Optimal Management of Basal Cell Carcinomas. Clinical Drug Investigation. Springer International Publishing; 2018 Aug 20;38(10):883-99.

33. Kyndi M, Sørensen FB, Knudsen H, Overgaard M, Nielsen HM, Andersen J, et al. Tissue microarrays compared with whole sections and biochemical analyses. A subgroup analysis of DBCG 82 b\&c. Acta Oncol. Taylor \& Francis; 2008;47(4):591-9.

34. Rizzardi AE, Zhang X, Vogel RI, Kolb S, Geybels MS, Leung Y-K, et al. Quantitative comparison and reproducibility of pathologist scoring and digital image analysis of estrogen receptor $\beta 2$ immunohistochemistry in prostate cancer. Diagn Pathol. 2016 Jul 11;11(1):914 11.

35. Knol J, Keller DS. Total Mesorectal Excision Technique-Past, Present, and Future. Clinics in Colon and Rectal Surgery. Thieme Medical Publishers; 2020 Apr 28;33(03):134-43.

36. Gouvas N, Agalianos C, Papaparaskeva K, Perrakis A, Hohenberger W, Xynos E. Surgery along the embryological planes for colon cancer: a systematic review of complete mesocolic excision. Int J Colorectal Dis. Springer Berlin Heidelberg; 2016 Sep;31(9):1577-94.

37. Yang L, Xiong Z, Xie Q, He W, Liu S, Kong P, et al. Prognostic value of total number of lymph nodes retrieved differs between left-sided colon cancer and right-sided colon cancer in 
stage III patients with colon cancer. BMC Cancer. BioMed Central; 2018 May 11;18(1):55812.

38. Sluijter CE, van Lonkhuijzen LRCW, van Slooten H-J, Nagtegaal ID, Overbeek LIH. The effects of implementing synoptic pathology reporting in cancer diagnosis: a systematic review. Virchows Arch. Springer Berlin Heidelberg; 2016 Apr 21;468(6):639-49.

39. Derwinger K, Carlsson G, Gustavsson B. Stage migration in colorectal cancer related to improved lymph node assessment. European Journal of Surgical Oncology (EJSO). 2007 Sep;33(7):849-53.

\section{Figure legends}

Figure 1 Images of TLR3, TLR5, TLR7, and TLR9 immunohistochemistry stainings representing colorectal cancer tumors with mild $(\mathbf{a}, \mathbf{c}, \mathbf{e}, \mathbf{g})$ and strong $(\mathbf{b}, \mathbf{d}, \mathbf{f}, \mathbf{h})$ staining. Images $\mathrm{a}-\mathrm{b}$ stained with a TLR3 polyclonal antibody, images $\mathrm{c}-\mathrm{d}$ stained with a TLR5 monoclonal antibody, images $\mathrm{e}-\mathrm{f}$ stained with a TLR7 polyclonal antibody, and images $\mathrm{g}-\mathrm{h}$ stained with a TLR9 polyclonal antibody. Original magnification: 20x.

Figure 2 Disease-specific survival analysis of different TLRs in colorectal cancer patients using the Kaplan-Meier method. (a) TLR3, (b) TLR5, (c) TLR7, and (d) TLR9. The log-rank test was used.

Figure 3 Disease-specific survival analysis of colorectal cancer patients using the Kaplan-Meier method. A mild versus strong (a) TLR5 expression among all patients, (b) TLR7 expression among all patients, (c) TLR5 expression among Dukes B patients, and (d) TLR5 expression among left-sided tumor patients. The log-rank test was used.

Supplementary Figure 1 Disease-specific survival analysis of colorectal cancer patients using the Kaplan-Meier method. (a) TLR5 expression among all patients, (b) TLR7 expression among all patients. The log-rank test was used. 


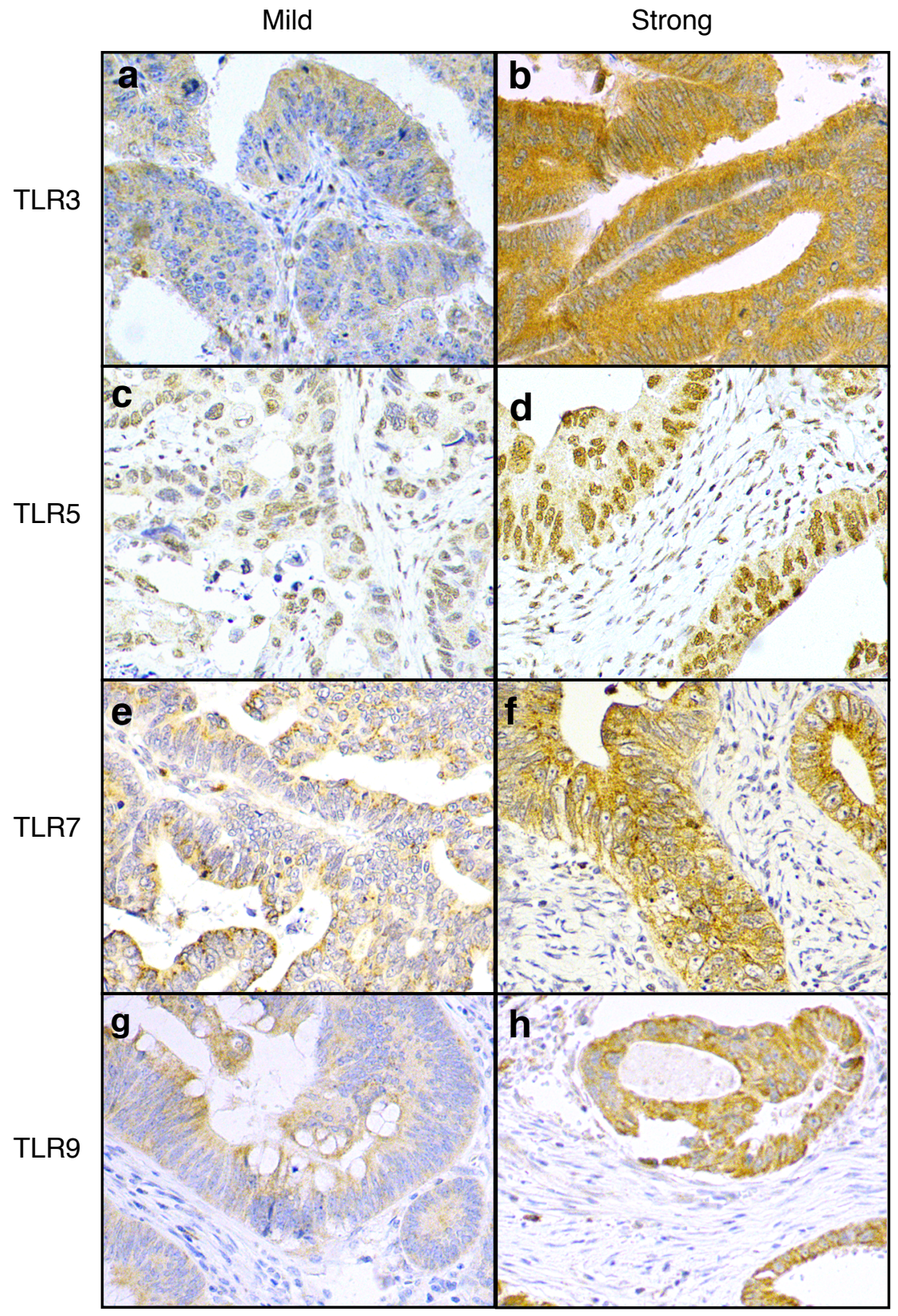

Figure 1 
a

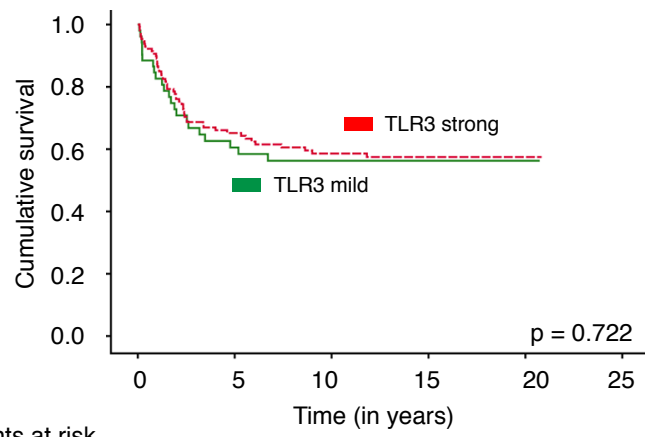

Patients at risk b

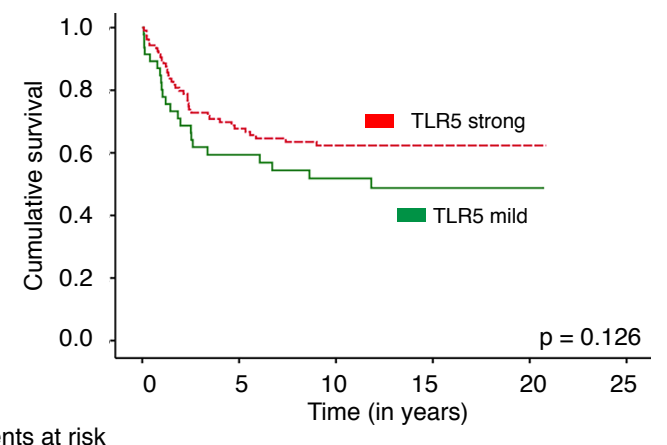

mild:

$143 \quad 85 \quad 59$
C

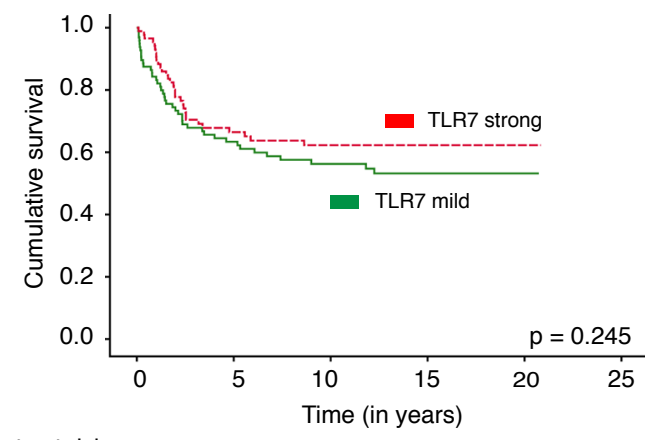

Patients at risk

TLR7 mild:

TLR7 strong: $\quad \begin{array}{lll}102 & 56 & 43\end{array}$ d

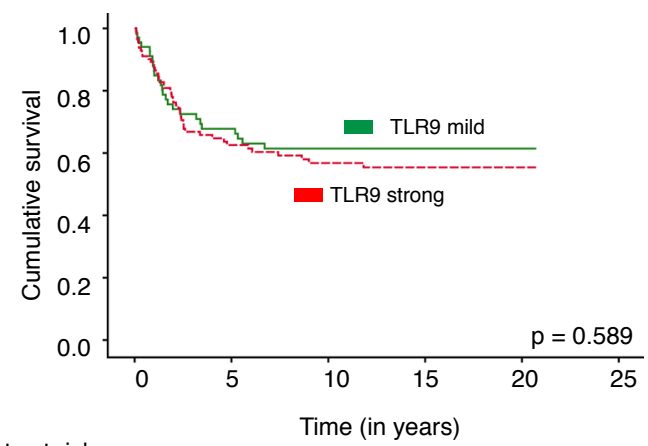

Patients at risk

$\begin{array}{lcccccc}\text { TLR9 mild: } & 71 & 43 & 31 & 28 & 7 & 0 \\ \text { TLR9 strong: } & 123 & 58 & 43 & 33 & 10 & 0\end{array}$

Figure 2 
a

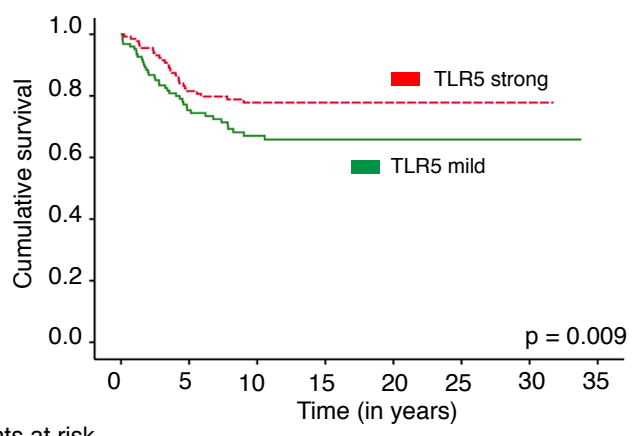

Patients at risk

$\begin{array}{lllllllll}\text { TLR5 mild: } \quad 363 & 177 & 126 & 100 & 64 & 22 & 4 & 0\end{array}$ TLR5 strong: $\begin{array}{llllllll}371 & 194 & 147 & 117 & 72 & 19 & 2 & 0\end{array}$

C

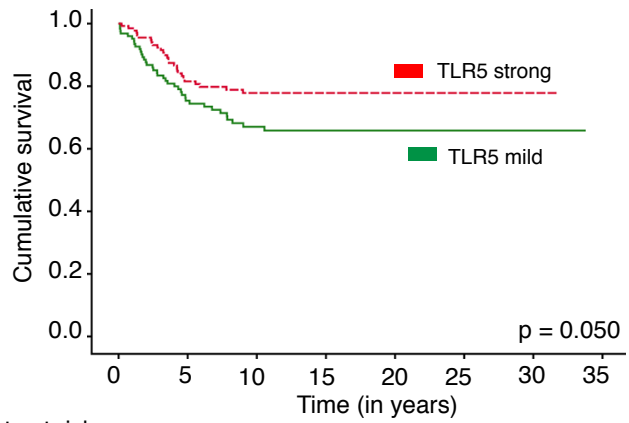

Patients at risk

$\begin{array}{lllllllll}\text { TLR5 mild: } \quad 130 & 82 & 56 & 42 & 23 & 7 & 2 & 0\end{array}$

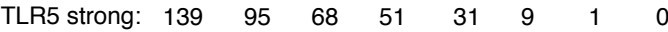

b

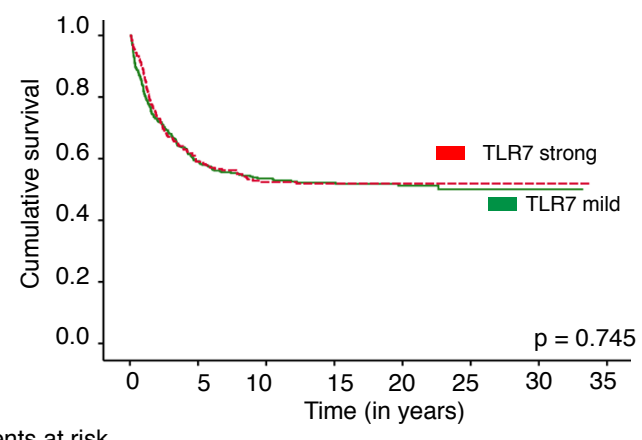

Patients at risk

$\begin{array}{lrrrrrrrr}\text { TLR7 mild: } & 466 & 236 & 166 & 128 & 81 & 23 & 4 & 0 \\ \text { TLR7 strong: } & 303 & 150 & 116 & 96 & 55 & 17 & 3 & 0\end{array}$

d

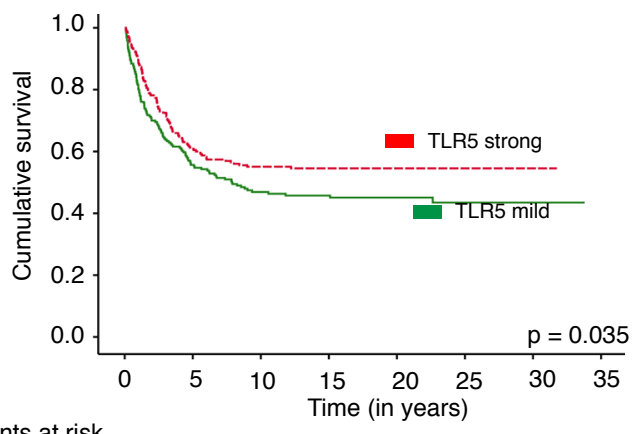

Patients at risk

$\begin{array}{lllllllll}\text { TLR5 mild: } \quad 299 & 147 & 106 & 87 & 56 & 17 & 5 & 0\end{array}$

$\begin{array}{llllllll}\text { TLR5 strong: } 354 & 186 & 143 & 117 & 112 & 71 & 2 & 0\end{array}$

Figure 3 


\section{Supplementary Figure 1}

a

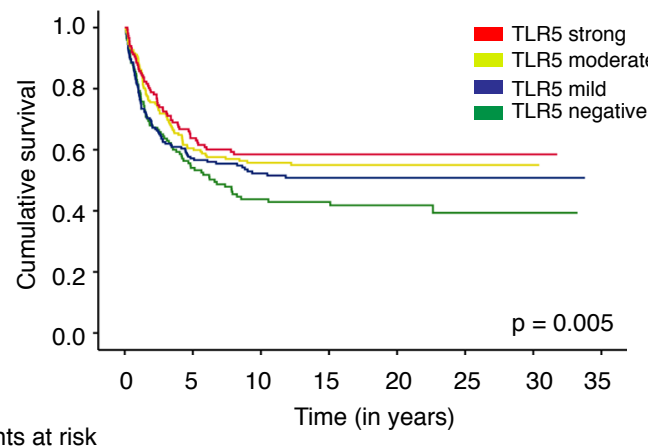

Patients at risk

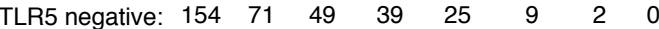
$\begin{array}{lllllllll}\text { TLR5 mild: } \quad 209 & 106 & 77 & 61 & 39 & 13 & 4 & 0\end{array}$

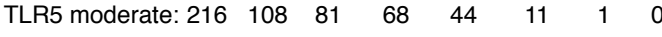

$\begin{array}{lllllllll}\text { TLR5 strong: } & 155 & 86 & 66 & 48 & 28 & 8 & 1 & 0\end{array}$ b

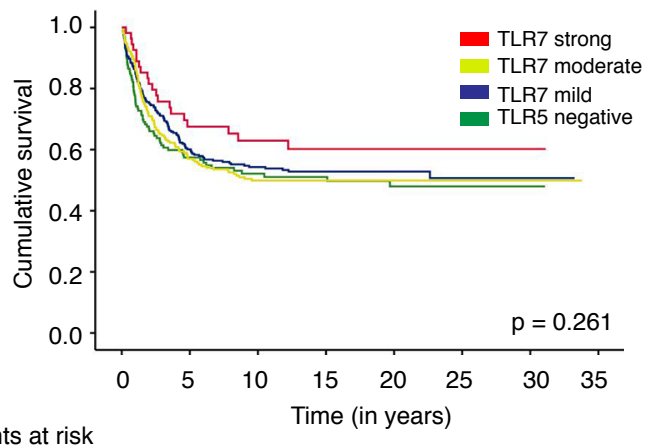

Patients at risk

$\begin{array}{lllllllll}\text { TLR7 negative: } 148 & 71 & 48 & 39 & 27 & 10 & 2 & 0\end{array}$ $\begin{array}{lllllllll}\text { TLR7 mild: } \quad 318 & 165 & 118 & 89 & 54 & 13 & 1 & 0\end{array}$ TLR7 moderate: $\begin{array}{llllllll}143 & 118 & 91 & 78 & 48 & 15 & 2 & 0\end{array}$

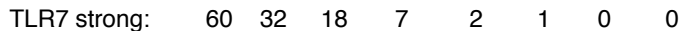


Supplementary Table 1. Clinicopathological characteristics of 825 colorectal cancer patients

\begin{tabular}{|c|c|}
\hline $\begin{array}{l}\text { Clinicopathological } \\
\text { variable }\end{array}$ & n (\%) \\
\hline \multicolumn{2}{|l|}{ Age } \\
\hline$<65$ & $352(42.5)$ \\
\hline$\geq 65$ & $474(57.5)$ \\
\hline \multicolumn{2}{|l|}{ Gender } \\
\hline Male & $457(55.4)$ \\
\hline Female & $368(44.6)$ \\
\hline \multicolumn{2}{|l|}{ Dukes stage } \\
\hline A & $122(14.8)$ \\
\hline $\mathrm{B}$ & $292(35.4)$ \\
\hline $\mathrm{C}$ & $225(27.3)$ \\
\hline $\mathrm{D}$ & $186(22.5)$ \\
\hline \multicolumn{2}{|l|}{ Grade (WHO) } \\
\hline 1 & $26(3.2)$ \\
\hline 2 & $563(68.4)$ \\
\hline 3 & $201(24.4)$ \\
\hline 4 & $33(4.0)$ \\
\hline \multicolumn{2}{|l|}{ Location } \\
\hline Colon & $424(51.4)$ \\
\hline Rectum & $401(48.6)$ \\
\hline \multicolumn{2}{|l|}{ Side } \\
\hline Right & $225(27.3)$ \\
\hline Left & $600(72.7)$ \\
\hline \multicolumn{2}{|l|}{ Histological type } \\
\hline Adenocarcinoma & $734(89.1)$ \\
\hline Mucinous & $90(10.9)$ \\
\hline
\end{tabular}


Table 1. Distribution of immunoactivity of TLRs among colorectal cancer patients

\begin{tabular}{|c|c|c|c|c|}
\hline & Negative (\%) & Low (\%) & Moderate (\%) & Strong $(\%)$ \\
\hline \multicolumn{5}{|c|}{ Pilot series $(\mathrm{n}=205)$} \\
\hline TLR3 & $1(0.5)$ & $54(27.3)$ & $125(63.1)$ & $19(9.1)$ \\
\hline TLR5 & $9(5.4)$ & $41(24.7)$ & $58(34.9)$ & $58(34.9)$ \\
\hline TLR7 & $18(9.0)$ & $84(42.2)$ & $75(37.7)$ & $22(11.1)$ \\
\hline TLR9 & $0(0.0)$ & $72(36.7)$ & $118(60.2)$ & $6(3.1)$ \\
\hline \multicolumn{5}{|c|}{ Whole cohort $(\mathrm{n}=825)$} \\
\hline TLR5 & $154(21.0)$ & $209(28.5)$ & $216(29.4)$ & $155(21.1)$ \\
\hline TLR7 & $148(19.2)$ & $318(41.4)$ & $243(31.6)$ & $60(7.8)$ \\
\hline
\end{tabular}


Table 2. Associations between TLR5 and TLR7 immunointensity and clinicopathological variables in 825 colorectal cancer patients

\begin{tabular}{|c|c|c|c|c|c|c|}
\hline \multirow{2}{*}{$\begin{array}{l}\text { Clincopathological } \\
\text { variable }\end{array}$} & \multicolumn{3}{|c|}{ TLR5 } & \multicolumn{3}{|c|}{ TLR7 } \\
\hline & Mild & Strong & $p$ value $^{1}$ & Mild & Strong & $p$ value $^{1}$ \\
\hline \multicolumn{7}{|l|}{ Age } \\
\hline$<65$ & $155(48.9)$ & $162(51.1)$ & 0.823 & $193(59.2)$ & $133(40.8)$ & 0.503 \\
\hline$\geq 65$ & 208 (49.9) & $209(50.1)$ & & $273(61.6)$ & $170(38.4)$ & \\
\hline \multicolumn{7}{|l|}{ Gender } \\
\hline Female & $161(49.7)$ & $163(50.3)$ & 0.941 & $271(62.4)$ & $163(37.6)$ & 0.235 \\
\hline Male & $202(49.3)$ & $208(50.7)$ & & $195(58.2)$ & $140(41.8)$ & \\
\hline \multicolumn{7}{|l|}{ Side } \\
\hline Right & $111(54.1)$ & $94(45.9)$ & 0.119 & $141(66.2)$ & $72(33.8)$ & 0.058 \\
\hline Left & $252(47.6)$ & $277(52.4)$ & & $325(58.5)$ & $231(41.5)$ & \\
\hline \multicolumn{7}{|l|}{ Location } \\
\hline Colon & $205(52.6)$ & $185(47.4)$ & 0.076 & $242(60.7)$ & $157(39.3)$ & 0.975 \\
\hline Rectum & $158(45.9)$ & $186(54.1)$ & & $224(60.5)$ & $146(39.5)$ & \\
\hline \multicolumn{7}{|l|}{ Histological type } \\
\hline Adenocarcinoma & $299(45.8)$ & $354(54.2)$ & $<0.001$ & $406(59.1)$ & $281(40.9)$ & 0.022 \\
\hline Mucinous & $63(78.8)$ & $17(21.3)$ & & $59(72.8)$ & $22(27.2)$ & \\
\hline \multicolumn{7}{|l|}{ Dukes stage } \\
\hline A & $46(43.0)$ & $61(57.0)$ & 0.388 & 77 (70.6) & $32(29.4)$ & 0.048 \\
\hline B & $130(48.3)$ & $139(51.7)$ & & $153(55.4)$ & $123(44.6)$ & \\
\hline $\mathrm{C}$ & $103(52.3)$ & $94(47.7)$ & & $128(60.7)$ & $83(39.3)$ & \\
\hline $\mathrm{D}$ & $84(52.2)$ & $77(47.8)$ & & $108(62.4)$ & $65(37.6)$ & \\
\hline \multicolumn{7}{|l|}{ Tumor grade (WHO) } \\
\hline $1-2$ & $251(47.6)$ & $276(52.4)$ & 0.118 & $297(54.1)$ & $252(45.9)$ & $<0.001$ \\
\hline $2-3$ & $112(54.4)$ & $94(45.6)$ & & $167(76.6)$ & $51(23.4)$ & \\
\hline
\end{tabular}

Abbreviations: TLR, toll-like receptor

${ }^{1}$ Chi-square test 
Table 3. Univariate and multivariate Cox regression analyses for disease-specific survival among colorectal cancer patients

\begin{tabular}{|c|c|c|c|c|c|c|}
\hline \multirow{3}{*}{ Age } & \multicolumn{3}{|c|}{ Univariate analysis } & \multicolumn{3}{|c|}{ Multivariate analysis } \\
\hline & \multirow{2}{*}{ HR } & \multirow[t]{2}{*}{$95 \% \mathrm{CI}$} & \multirow[t]{2}{*}{$p$ value } & \multirow[t]{2}{*}{ HR } & \multirow[t]{2}{*}{$95 \%$ CI } & \multirow[t]{2}{*}{$p$ value } \\
\hline & & & & & & \\
\hline$<65$ & 1.00 & & & 1.00 & & \\
\hline$\geq 65$ & 1.02 & $1.01-1.02$ & 0.004 & 1.03 & $1.02-1.05$ & $<0.001$ \\
\hline \multicolumn{7}{|l|}{ Gender } \\
\hline Female & 1.00 & & & 1.00 & & \\
\hline Male & 0.96 & $0.78-1.18$ & 0.706 & 1.05 & $0.84-1.31$ & 0.680 \\
\hline \multicolumn{7}{|l|}{ Dukes stage } \\
\hline A & 1.00 & & & 1.00 & & \\
\hline $\mathrm{B}$ & 2.18 & $1.29-3.69$ & 0.004 & 2.15 & $1.22-3.78$ & 0.008 \\
\hline $\mathrm{C}$ & 5.29 & $3.17-8.82$ & $<0.001$ & 6.07 & $3.50-10.5$ & $<0.001$ \\
\hline $\mathrm{D}$ & 38.3 & $21.4-68.5$ & $<0.001$ & 46.2 & $24.7-86.6$ & $<0.001$ \\
\hline \multicolumn{7}{|c|}{ Tumor grade (WHO) } \\
\hline $1-2$ & 1.00 & & & 1.00 & & \\
\hline $3-4$ & 2.48 & $1.80-3.42$ & $<0.001$ & 2.38 & $1.69-3.35$ & 0.001 \\
\hline \multicolumn{7}{|l|}{ Location } \\
\hline Colon & 1.00 & & & 1.00 & & \\
\hline Rectum & 0.78 & $0.59-1.04$ & 0.094 & 1.15 & $0.72-1.34$ & 0.906 \\
\hline \multicolumn{7}{|l|}{ TLR5 } \\
\hline Mild & 1.00 & & & 1.00 & & \\
\hline Strong & 0.75 & $0.60-0.94$ & 0.011 & 0.74 & $0.59-0.92$ & 0.007 \\
\hline \multicolumn{7}{|l|}{ TLR7 } \\
\hline Mild & 1.00 & & & & & \\
\hline Strong & 0.97 & $0.78-1.20$ & 0.754 & & & \\
\hline
\end{tabular}

Multivariate analysis included adjustments for Dukes stage, tumor grade, and tumor location.

Abbreviations: TLR, toll-like receptor, CI, confidence interval; HR, hazard ratio 
Table 4. Survival analysis by subgroups, strong TLR5 and TLR7 expression compared strong to mild, in 825 colorectal cancer patients

\begin{tabular}{|c|c|c|c|c|c|c|}
\hline & \multicolumn{3}{|c|}{ Strong TLR5 } & \multicolumn{3}{|c|}{ Strong TLR7 } \\
\hline & HR & 95\% CI & $p$ value & HR & $95 \% \mathrm{CI}$ & $p$ value \\
\hline \multicolumn{7}{|l|}{ Age } \\
\hline$<65$ & 0.88 & $0.63-1.24$ & 0.462 & 1.10 & $0.75-1.48$ & 0.768 \\
\hline$\geq 65$ & 0.67 & $0.50-0.90$ & 0.007 & 0.92 & $0.69-1.23$ & 0.557 \\
\hline \multicolumn{7}{|l|}{ Gender } \\
\hline Female & 0.71 & $0.51-0.99$ & 0.042 & 1.19 & $0.86-1.64$ & 0.1301 \\
\hline Male & 0.79 & $0.59-1.10$ & 0.119 & 0.80 & $0.59-1.09$ & 0.161 \\
\hline \multicolumn{7}{|l|}{ Dukes stage } \\
\hline A & 0.55 & $0.20-1.55$ & 0.257 & 0.18 & $0.02-1.36$ & 0.096 \\
\hline $\mathrm{B}$ & 0.61 & $0.37-1.00$ & 0.050 & 0.96 & $0.59-1.54$ & 0.856 \\
\hline $\mathrm{C}$ & 0.96 & $0.64-1.42$ & 0.821 & 1.11 & $0.75-1.64$ & 0.604 \\
\hline $\mathrm{D}$ & 0.76 & $0.54-1.06$ & 0.104 & 0.89 & $0.64-1.24$ & 0.500 \\
\hline \multicolumn{7}{|l|}{ Tumor grade (WHO) } \\
\hline $1-2$ & 0.76 & $0.59-1.01$ & 0.062 & 1.01 & $0.78-1.31$ & 0.934 \\
\hline $3-4$ & 0.76 & $0.52-1.12$ & 0.167 & 1.08 & $0.70-1.68$ & 0.720 \\
\hline \multicolumn{7}{|l|}{ Location } \\
\hline Colon & 0.82 & $0.60-1.21$ & 0.213 & 1.06 & $0.78-145$ & 0.708 \\
\hline Rectum & 0.67 & $0.49-0.92$ & 0.012 & 0.87 & $0.64-1.20$ & 0.396 \\
\hline \multicolumn{7}{|l|}{ Histological type } \\
\hline Adenocarcinoma & 0.73 & $0.58-0.93$ & 0.010 & 0.91 & $0.72-1.15$ & 0.435 \\
\hline Mucinous & 1.25 & $0.61-2.55$ & 0.544 & 1.88 & $0.98-3.60$ & 0.058 \\
\hline
\end{tabular}

Abbreviations: TLR, toll-like receptor, CI, confidence interval, HR, hazard ratio 